\title{
Paolo e Francesca (1950) e il cinema drammatico di Raffaello Matarazzo
}

\author{
Gino Frezza \\ Università di Salerno \\ frezza@unisa.it
}

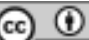

\begin{abstract}
Il cinema di Raffaello Matarazzo è noto soprattutto per il grande successo ottenuto, negli anni cinquanta, dai suoi melodrammi popolari (Catene, Tormento, I figli di Nessuno, L'Angelo bianco ecc.). Ma nel 1950, prima di Catene, il grande regista italiano dirige un film tratto dalla vicenda di Paolo e Francesca. Qual è il rapporto fra la visione filmica di Matarazzo e il V canto dell'Inferno di Dante? Il saggio di Frezza puntualizza il quadro del cinema italiano dell'immediato secondo dopoguerra, confronta il film di Matarazzo con il coevo Il conte Ugolino diretto nel 1949 da Riccardo Freda e, infine, indaga le modalità con cui Matarazzo formula un modello di "dramma filmico" che costituisce un'attenta, strategica, riformulazione audiovisiva del testo dantesco.

Parole chiave: cinema italiano e secondo dopoguerra; Dante e il film sonoro; soggetto e plot narrativo; dramma filmico.
\end{abstract}

\begin{abstract}
Raffaello Matarazzo's cinema is known for the great success, in the fifties, of its popular melodramas (Catene, Tormento, I figli di Nessuno, L'Angelo bianco, etc.) But in 1950, before Catene, the great Italian filmmaker directed a film based on the story of Paolo and Francesca. What is the relationship between the cinematic vision of Matarazzo and the fifth canto of Dante's Inferno? Frezza's essay approaches the bakground of Italian's cinema during the immediate post-war period, and compares Matarazzo's film with Il conte Ugolino directed in I949 by Riccardo Freda. Finally it looks at the ways in which Matarazzo formulates a "filmic drama model" which is a careful and strategic audiovisual reformulation of Dante's text.
\end{abstract}

Keywords: italian cinema and second post-war period; Dante and sound film; subject and narrative ploy; film drama. 


\section{Sul cinema di Raffaello Matarazzo}

$\mathrm{D}$ i Raffaello Matarazzo oggi quasi nessuno scrive o tratta delle opere filmiche da lui dirette. Sempre di meno se ne parla, a differenza degli anni Settanta, quando invece si mobilitò una parte allora "giovane" della critica e della storiografia italiana del cinema, per restituire a questo importante regista il senso e il valore dei contributi forniti al cinema italiano del secondo dopoguerra (Aprà et alii 1976; Aprà e Carabba 1976; sul "caso" Matarazzo torna, più recentemente, Della Casa 2000: 783-784). Si rimarcò, peraltro, come egli avesse svolto un ruolo cruciale nell'ambito della stagione degli anni trenta, risultando non soltanto anticipatore del neorealismo (come in Treno popolare del I933), ma altresì autore di vivaci e ben congegnate commedie che rappresentavano in modo assolutamente originale vari caratteri della borghesia italiana.

Lattenzione critica verso il cinema di Matarazzo in buona parte si concentrò, tuttavia, su quello che venne definito, sbrigativamente e forse approssimativamente, "neorealismo d'appendice": si tratta di quel gruppo di opere che da Catene, uscito nel I950, e per quasi tutto il decennio successivo, da Tormento a I figli di Nessuno, Vortice, L'angelo bianco, fino a Malinconico autunno, del 1958, marcò l'immaginario popolare italiano, con un successo al botteghino tale da consentire a una casa di produzione come la Titanus di salvarsi da una crisi finanziaria. La critica e la storiografia degli anni Settanta non soltanto ricostruirono attentamente il dibattito critico-ideologico che questa tipologia di film di Matarazzo produsse nella cultura italiana degli anni Cinquanta, ma contestualmente riconobbero al suo interno la tessitura di un moderno linguaggio espressivo, uno sguardo narrativo assai lucido e una grande maestria nel "trattare" la materia sentimentale, anche se attraverso intrecci narrativi che ripescavano la tradizione del feuilleton ottocentesco e lo riadattavano alla fase storica del passaggio, non indolore nel nostro paese (specie al Sud), da una società prevalentemente contadina a una moderna e industriale (si trattava, dunque, di storie riguardanti padri o madri separati dai loro figli, spesso accusati da una giustizia cieca, vittime di soprusi o tradimenti non casualmente innescati nell'ambito familiare, di agnizioni e riconoscimenti a distanza di anni, di tormenti e sofferenze subite silenziosamente ma dignitosamente, ecc. ecc.).

Oggi, a distanza di quattro decenni dal periodo in cui si ridiscusse e si riesaminò la filmografia di Matarazzo, sembra che tutto sia passato senza lasciare alcun segno; al massimo si riconosce quasi istituzionalmente l'operazione culturale di Matarazzo in un decennio di forti trasformazioni sociali, ma non si ravvisano conseguenze di tipo teorico e storico, per esempio nel re-inquadrare il valore attuale che quel cinema può, semmai, ancora esprimere; ne varrebbe forse la pena, anche se la situazione odierna - l'era delle comunicazioni digitali per le quali il cinema stesso è posto radicalmente in questione, nel suo ruolo di 
medium e nelle sue capacità di segnare la comunicazione collettiva - è incomparabile sia nei confronti degli anni Cinquanta del XX secolo (marcati dallo scarto fra la ricostruzione dalle macerie della Seconda Guerra e l'imminenza del boom economico), sia in relazione agli anni Settanta (a loro volta segnati da un dissidio, ancor poco riconciliato, fra cinema e televisione).

Il fatto, dunque, che nella filmografia di Raffaello Matarazzo sia presente un titolo come Paolo e Francesca, ispirato alla ben nota vicenda degli amanti trattata nel V Canto della Commedia dantesca, film realizzato nel 1950, ossia nella fase pienamente sonora del cinema, in un periodo in cui ancora non è emersa la centralità della televisione pubblica e il cinema si avvale della piena articolazione delle risorse tecnico-espressive che fanno i conti, da un lato col neorealismo e, dall'altro, con lo sviluppo di un sistema industriale filmico avanzato, può essere vivo spunto per rilanciare l'attenzione verso il cinema di Matarazzo. Un regista che non soltanto dimostra, anche in questo caso, di possedere e mettere a frutto capacità creative di grande professionalità ma, per di più, di saper esprimere una irriducibile idea del cinema. Che si può sintetizzare con la formula: ogni storia raccontata sullo schermo si definisce quale spazio narrativo prossimo alla tragedia, soltanto in questo modo il medium esibisce un potente effetto catartico, dissimulando e, insieme, portando alla luce il rapporto drammatico fra desiderio, immaginario e realtà.

Nella filmografia di Matarazzo Paolo e Francesca precede esattamente Catene $^{\mathrm{I}}$ e dunque si pone praticamente al di qua di quel filone del cinema melodrammatico che rese il regista molto discusso nella cultura italiana dell'epoca. Nella parte di Francesca da Rimini, l'attrice francese Odile Versois, nella parte di Paolo Malatesta l'attore italiano Armando Francioli, mentre le parti di Gianciotto Malatesta e del suo fido astrologo di corte sono rispettivamente interpretate da Andrea Checchi e Aldo Silvani.

Prima ancora di affrontare la questione della relazione fra questo film sonoro e il V canto dell'Inferno di Dante, occorre chiedersi perché Raffaello Matarazzo scelga di dirigere questo film. La risposta non è semplice, anche perché non si rintracciano fonti critiche o testimonianze significative al riguardo. Nei materiali storico-critici esistenti che riguardano questo regista italiano, quasi tutti riguardanti la stagione del melodramma popolare, e in quelle scarse testimonianze relative all'opera del regista (romano, ma di origini napoletane), i cenni relativi a questo film si riducono a quasi nulla. I pochi giudizi - talvolta dello stesso regista - sono diminutivi e l'opera viene quasi sempre messa da parte, come almeno incompiuta o non riuscita. ${ }^{2}$

I. Così è indicato nella filmografia contenuta in Aprà et alii (Aprà et alii 1976: 28); il database IMDB, invece, indica Paolo e Francesca come parentesi fra Catene - attribuito al 1949 - e Tormento, collocato, nel I950, di seguito al dramma tratto da Dante.

2. In una intervista concessa a Bernard Eisenschitz nel 1964, Matarazzo risponde, a una domanda 
Eppure oggi, rivedendolo accuratamente, il film risulta degno di interesse e può rischiarare un momento importante della storia del nostro cinema, anche in riferimento al tentativo di offrire agli spettatori una versione credibile dei personaggi e della vicenda riferiti al canto dantesco.

\section{Il CONTESTO DEL FILM D'AUTORE E DEL FILM DI GENERE IN ITALIA NEL I950}

Nella fase assai viva e caotica dell'immediato secondo dopoguerra, il cinema italiano riprende quel ruolo centrale già conquistato nel panorama del cinema internazionale, e giunto a un punto di forzata irresoluzione nel corso dei tardi anni trenta e nei primi quaranta. Sia il fascismo sia la gestione politica dell'istituzione filmica, in particolare la censura, non riescono infatti a impedire che il cinema italiano, durante la seconda guerra, affermi una indiscutibile vitalità, ponendo alcune solide basi alla rinascita del dopoguerra. L'affermazione critica e teorica del neorealismo dal 1945 in poi, aggiunge un tassello decisivo, una pietra di paragone importante e affermativa della coscienza che il cinema italiano assume davanti al compito di delineare un immaginario moderno, riconoscibile nelle capacità di squadernare i lati anche più nascosti del reale, ma altresì nelle qualità spettacolari ed espressive. Il cinema italiano dell'immediato secondo dopoguerra mostra un complesso scenario, al cui interno le istanze neorealiste fanno i conti con l'emergere di una società che progressivamente intende riconoscersi nella pienezza di un immaginario e, contemporaneamente, in tutti gli estremi possibili - ma anche nei lati "medi" - del narrabile e del visualizzabile. E così, assieme alla maturazione dei capolavori del neorealismo (fenomeno a sua volta stratificato e dalle diverse linee creative implicate, di straordinario valore e risonanza culturale ma produttivamente minoritario), nel quadro generale del cinema si affermano, come preminenti, le linee produttive del film di genere (iniziando dal film comico), all'interno delle quali si tentano le più diverse strade per focalizzare e soddisfare la domanda proveniente da diversi pubblici di spettatori. Dal film d'avventura al cappa e spada, dal film storico al film-opera, dalla parodia al film musicale, al film di guerra ecc.

Attorno al 1950, si situa una specie di giro di boa, come una riconversione complessiva delle istanze narrative/creative emerse nella fase dei precedenti cinque anni. Diverse testimonianze sulla chiusura, o sulla interna trasformazione, della stagione del neorealismo raccontano (talvolta in cifra, ma talora chiaramente) come il cinema italiano stia delineando nuove possibilità, ma-

relativa al film e alla sua inclusione nell'ambito del genere storico, con questo lapidario giudizio:

"Non credo che fosse riuscito. C'era un budget piccolissimo" (in, Aprà et alii 1976: 84). 
turate anche nell'agone di uno sviluppo non sempre armonico, se non diseguale e talvolta contrastato (sul dibattito tra fine degli anni Quaranta e primi Cinquanta, fra cinema e letteratura, attorno a neorealismo e post-neorealismo: AA.VV. 1980; Parigi 20I4: 269-302. Sulla configurazione generale, economica e culturale, del cinema nel dopoguerra e sulla complessità del rapporto fra offerta di cinema e pubblici: AA.VV. 1985, Magrelli 1986, Zagarrio 1988, Frezza 1996, Casetti e Fanchi 2006, Casetti e Mosconi 2006). In tale quadro, il film di genere manifesta una serie di valenze utili sia produttivamente sia in termini di risposta all'immaginario attuale: da un lato "lavora" la sostanza, consolidata, di stereotipi ben accettati, che edificano il consenso del vasto pubblico, dall'altro non impedisce la ricerca di variazioni e sperimentazioni che alimentino la curiosità del pubblico intelligente; questo può accadere, e accade, specialmente in un tessuto produttivo come quello italiano, che presenta vari margini aleatori e una serializzazione di basso-medio regime. Così, la notazione d'autore e la sperimentazione di alcune novità espressive si manifestano chiaramente nel film di genere italiano: basti pensare a certi film comici di Steno-Monicelli, ai drammi di Pietro Germi o di Gianni Franciolini, al primo Lattuada, a un autore chiave (ancora oggi da rivalorizzare pienamente) del film "medio" italiano, e tuttavia innovativo, come Mario Soldati, a un regista di spicco per ogni tipo di "genere" filmico come Riccardo Freda (su cui tornerò fra poco), all'opera fuori schema di un regista anticipatore come Vittorio Cottafavi. E tutto ciò, senza neanche voler considerare lo spazio occupato in modo assai vivace dai bravi professionisti italiani dei generi del cinema, che gettano un ponte davvero significativo fra l'esperienza degli anni trenta e il dopoguerra, come Mario Mattoli, Giacomo Gentilomo, Carlo Ludovico Bragaglia, Camillo Mastrocinque.

Fra queste figure, Riccardo Freda diviene estremamente interessante per la nostra analisi di Paolo e Francesca di Matarazzo, per diversi motivi. Anzitutto i due registi si conoscevano bene ed erano molto amici (Freda, prima di esordire come regista, era stato produttore di alcuni film di Matarazzo). Alle memorie di un Freda anziano (Freda 198I) si devono alcune scarne valutazioni elogiative sull'opera dell'amico (in genere, diversi colleghi riconoscono a Matarazzo grande professionalità e cultura, ma senza mai entrare nel merito del suo lavoro di regia; Freda al contrario gli restituisce quello che altri non gli hanno mai inteso concedere...). E, all'esame delle rispettive filmografie, non può non essere notato che nel 1949, ossia un anno (o quasi) prima di Paolo e Francesca, lo stesso Freda dirige Il conte Ugolino, altro titolo evidentemente riferito all'opera dantesca. 


\section{DAL MUTO AL SONORO}

Qualcuno potrà ritenere che l'uscita quasi contemporanea dei due film possa riaprire il discorso su una possibile "tradizione di film danteschi". Ma per dare senso a una tradizione di questo genere occorrerebbe dimostrare, senza dubbi, che di vera tradizione si tratti, piuttosto che di un elenco - più o meno lungo, da collocare in relazione al numero complessivo dei film prodotti in un dato periodo, affinché se ne colga la rilevanza anche quantitativa - di pellicole riferite in maniera molto differenziata a parti dell'opera dantesca. Parlare di "tradizione" comporta infatti una uniformità di modi espressivi, una forma riconoscibile e un repertorio di elementi (dai temi narrativi alla struttura visiva delle inquadrature) che si ripetano e si ricollochino chiaramente, di là dal mero adattamento di parti, celebri o meno celebri, della Commedia. Ora, davvero ciò avviene nei film tratti da Dante? Altra questione da risolvere sarebbe in che senso, e come, una tale tradizione riuscirebbe a "integrare" i film prodotti nel periodo del cinema muto con quelli realizzati dopo l'avvento del sonoro. E qui, subito, si nota che, nel sonoro, l'elenco dei film riferiti a Dante si riduce esponenzialmente rispetto al numero dei film muti. Si aggiunge quindi una questione ulteriore: perché tale riduzione? Molte possono essere le spiegazioni utili. Una fra queste potrebbe basarsi sul concetto che, durante l'epoca del muto, s'incrementi la produzione di film tratti da Dante (almeno fino ai primi anni venti) in quanto è favorita una sorta di corrispondenza pregnante e cruciale fra scrittura letteraria e scrittura filmica (basti pensare al piano del dialogo e a quello della scrittura narrativa, e altresì di quella poetica, trasferiti su quello della didascalia filmica). In altri termini: la scrittura dantesca potrebbe essersi rivelata una specie di elemento facilitatore della definizione delle immagini, soprattutto quando queste siano (come le didascalie) immagini di scritture. Ė una ipotesi da verificare.

Ma altre spiegazioni potrebbero avere diverso orientamento; per esempio, non c'è dubbio che, sul piano storico, il film muto adopera una serie ricca di elementi culturali da rivivificare entro la nuova drammaturgia del medium e, fra questi, quelli appartenenti al vasto e ben conosciuto repertorio della letteratura in lingua italiana (naturalmente, Dante in primis) costituiscono un architrave fondamentale del consenso ottenuto presso il grande pubblico. Ancora: è l'immaginario complessivo italiano di primo Novecento - rielaborando la ripresa di elementi danteschi nelle arti pre-cinematografiche, da quelle visive alle musicali e teatrali - a rendere particolarmente significante il rapporto fra le narrazioni dell'inizio del XX secolo e le radici culturali del passato. Da qui, anche, la rilevanza sociologica del ritorno dell'opera di Dante in produzioni di cinema che dunque "lavorano" sul piano della memoria collettiva (riprendendo, talvolta, significative rielaborazioni poetiche-critiche sui 
personaggi della Commedia: su Paolo e Francesca, si ricordino le posizioni espresse da Boccaccio, De Sanctis, ecc.).

Ma, con l'avvento del sonoro, l'asse su cui il cinema muto può aver ricavato dal testo dantesco una serie di materiali sviluppabili nel racconto filmico si riformula radicalmente: le parole scritte e le didascalie divengono voci, incorporate in figure - poco importa che siano vive o spettrali o dissolventi nell'articolazione del montaggio - che agiscono e occupano il divenire dello spazio e del tempo dell'immagine in movimento, e i gesti divengono talvolta più netti e decisivi delle parole stesse. Con il cinema sonoro, ogni facile o diretta corrispondenza con le parole e la scrittura dissolve a fronte dell'imperativo di costruire uno spazio-tempo dell'immagine in grado di raccontare in piena autonomia. L'intero quadro delle possibilità di ritagliare la dinamica videosonora e di praticare feconde interazioni fra suoni, parole, musiche e immagini si rivela gradualmente, tra fase classica e fase moderna, non in modo univoco, non senza conflitti e differenziazioni estetico-creative, e secondo rapporti non determinati in partenza (Deleuze I984 e 1989).

Così il film ispirato o tratto da Dante diventa più difficile e "ostico" di quanto fosse nel periodo del muto. Occorrono modelli culturali in grado di affrontare la sfida di una visualizzazione "a tutto campo" e - tendenzialmente - senza limiti di possibilità, ma anche in grado di essere credibile nella dimensione storico-narrativa. In tal senso, il progetto di un cinema riferito all'autore della Commedia può essere sostenibile a patto che riesca a condensare più assi portanti: una dinamica storica in ogni senso resa chiara e sintetica, afferrabile sul piano scenografico e del disegno degli ambienti e dei costumi; l'evidenza di un dramma pubblico e privato in cui i conflitti fra personaggi siano argomentati con efficacia; un esito narrativo non scontato e non preventivamente risolto dal testo dantesco di partenza, seppure in un'ottica di trasposizione fedele che, tuttavia, raggiunga il senso "in più" da attribuire alla rappresentazione filmica.

Non c'è allora dubbio che una fase produttiva di tal rango maturi subito dopo la seconda guerra mondiale. In Italia il cinema viveva un momento di slancio produttivo e, in tale contesto, si situano i due film di Matarazzo e di Freda. Essi possono essere utilmente collocati su due estremi: tanto il primo, Paolo e Francesca, è concentrato a restituire il senso di un dramma sentimentale in una cornice storica resa con netta chiarezza di contorni, secondo gli stilemi di un modello intenso e tragico di melodramma agito entro la sfera familiare, in cui l'amore sincero fra due giovani viene impedito dalle circostanze e avvelenato dal sospetto; ${ }^{3}$ quanto il secondo, Il conte Ugolino, nel dipingere

3. La predilezione di Matarazzo per il melodramma è confermata non solo dai suoi grandi film "popolari", ma altresì da un titolo come Giuseppe Verdi, da lui diretto nel 1953: oggi si direbbe un biopic dedicato al grande compositore. 
un contesto storico medioevale, è focalizzato a una rappresentazione del conflitto di potere, entro la sfera pubblica, ossia oltre il dramma sentimentale, in una trama in cui l'inganno si contrappone alla buona fede e alla lealtà. ${ }^{4}$

Questi due film sonori in ogni caso attestano l'esistere di un momento produttivo in cui alcuni settori della produzione filmica italiana, fra la fine degli anni Quaranta e l'inizio del successivo Decennio, si sono posti il compito di rappresentare sullo schermo, usando le contemporanee tecnologie e competenze video-sonore, parti della Commedia dantesca che potessero conquistare l'attenzione del grande pubblico. Quanto una tale ipotesi possa essere confermata, è una questione che potrebbe essere risolta soltanto da un attento studio comparativo di diversi titoli del nostro cinema situabili in quello scorcio d'anni, alcuni ascrivibili al dramma storico, altri al panorama di racconti tratti da note opere letterarie (non solo italiane), e altri alla contaminazione (già in quel periodo emergente) fra il racconto storico e l'ambientazione avventurosa-fantastica. Si tratta di un compito qui esplorabile soltanto in minima parte, che potrebbe essere oggetto di un apposito programma di ricerca da condurre in gruppo.

Tuttavia l'analisi del solo Paolo e Francesca di Matarazzo può evidenziare il senso recondito per cui nel cinema italiano di quel periodo il riferimento a Dante viene assunto con forte consapevolezza dei limiti della trasposizione filmica e, assieme, della necessità di ricostruire un plot da collocare nell'ambito dell'universo dantesco, tentando una scommessa difficile: da un lato seguendo parametri espressivi condivisi dal pubblico, e dall'altro "giocando" un registro di tempi e di modalità di linguaggio con i quali raggiungere una propria autonomia di significati.

\section{LA QUESTIONE DEL SOGGETTO}

Si può dire, o no, che Paolo e Francesca diretto da Matarazzo è una trasposizione del V Canto dell' Inferno? Nella risposta che si dà a tale domanda è forse racchiuso il senso completo dell'operazione filmica realizzata nel 1950 dal nostro regista.

Chiunque sappia qualcosa di produzione di cinema narrativo ha ben presente che, per intraprendere un investimento produttivo, occorre primariamente risolvere - nella decisione di girare una storia di immagini, suoni e dialoghi - la questione del soggetto, ossia della storia e dei fatti da raccontare.

4. Gli sceneggiatori del film di Freda risultano essere Stefano Vanzina (ossia Steno, per il quale, con un mero refuso, i titoli iniziali del film riportano erroneamente il cognome Vanzini) e Mario Monicelli. I due grandi autori della commedia italiana degli anni '5o, all'inizio del decennio ancora "giovani" sceneggiatori e poco prima di porsi dietro la macchina da presa, dimostrano di essere anche intelligenti riscrittori del dramma storico-politico! 
Quindi, la domanda di sopra può essere riformulata in questi termini: il soggetto del film di Matarazzo è una trasposizione del relativo brano del poema dantesco?

Sono dell'avviso che il soggetto del film di Matarazzo ha a che fare col canto dantesco soltanto indirettamente, ed esclusivamente per quanto attiene alla parte finale, mentre in realtà il corpo generale del racconto filmico è riferito a una ricostruzione simil-storica, derivata da informazioni contestuali, di genere soprattutto storiografico, e, forse anche, para-testuali. La ragione di ciò è piuttosto semplice ed esplicabile grazie alle logiche fondative del linguaggio narrativo filmico. Dal punto di vista delle necessità di questa forma di cinema, è il V canto dell' Inferno ad essere (legittimamente sia chiaro!) scarsamente filmico, se non nella potenza evocativa della scrittura dantesca, la quale tuttavia non offre se non parchi segmenti - alcuni senz'altro fulminanti - di azione narrativa, ossia pochissime indicazioni di gesti e di snodi d'azione in grado di costituire e comporre una storia per lo schermo sonoro. Nel V Canto, semmai, l'azione consiste nell'introdurre il sommo poeta nel secondo Girone infernale, e solo nella parte finale del Canto si riconosce quasi un simulacro di racconto nella presupposizione di fatti che le parole di Francesca esprimono alla sensibilità del suo interlocutore (lo stesso Dante); tutto si esprime, in altri termini, nelle impressioni che il racconto di Francesca scatena nella sensibilità del poeta.

Si può dunque comprendere in che senso il film di Matarazzo non sia una corrispondente trasposizione filmica; il meccanismo espressivo del film narrativo (quello classico soprattutto, alla cui forma generale senźaltro appartiene il film di Matarazzo) implica uno sviluppo di premesse motivazionali, situazioni che consentano di incrociare e confrontare personaggi, notazioni d'ambiente, visualizzazione scenica, dinamiche di gesti e dialoghi contemporaneamente finalizzati a ritagliare le fisionomie dei personaggi implicati nella storia. Tenendo a mente tali esigenze di credibilità narrativa, gli sceneggiatori del film (Vittorio Calvino e Vittorio Nino Novarese, assieme allo stesso Matarazzo) hanno strutturato il plot: nel I250, dal conflitto armato di due città, Rimini e Ravenna (questa assediata dal signore della prima, Gianciotto Malatesta), scaturisce il fortuito incontro di Paolo Malatesta, fratello di Gianciotto, introdottosi nella città assediata per verificare come sabotare le provviste alimentari che prolungano la sopravvivenza dei nemici, e di Francesca, figlia del conte Guido di Ravenna, che opera da infermiera nell'ospedale messo in piedi dalle suore della cattedrale. Senza che si dicano i rispettivi nomi, e senza conoscere le posizioni che essi occupano nello scacchiere politico del conflitto, i due giovani vengono posti nella tipica situazione del soccorso d'amore: Paolo, riconosciuto spia, scappando dalla folla dei ravennati, ripara nella Cattedrale e viene tutelato dalla furia dei suoi inseguitori grazie all'intervento di Francesca, la quale 
favorisce il rientro del giovane, salvo, fuori le mura. I due si sono innamorati reciprocamente, ma silenziosamente, anzi disinteressatamente, ignari delle conseguenze dei loro gesti. E così, poco dopo, al momento in cui il nemico di Ravenna, Gianciotto, fratello di Paolo, toglie l'assedio tentando una soluzione diplomatica del conflitto, chiede in sposa, a garanzia del patto suggellato con l'ex nemico, la figlia del conte Guido. La giovane donna, alla notizia, dapprima si sente costretta dalla ragion di stato a sposare uno sconosciuto senza amore, poi - siccome Gianciotto invia il fratello Paolo a suggellare in sua vece il contratto politico e familiare con il suo ex nemico - riconosce in Paolo il giovane di cui è già innamorata e accetta di sposarlo; ben presto, però, la sua illusione svanisce, scoprendo che il suo sposo è piuttosto un altro! Francesca e Paolo, dunque, devono forzatamente accettare il fato sventurato che li ha costretti a star vicini ma a non potersi amare, mentre Lei deve compiere il suo dovere di sposa con Gianciotto, che ha una fisionomia e un carattere ben diversi da quelli di Paolo ed è, inoltre, claudicante dal piede sinistro. Ma l'astrologo vicino a Gianciotto, nelle funzioni di saggio consigliere del Signore di Rimini, ha visto quel che non doveva vedere, ossia l'amore inconfessabile dei due giovani, e così, tentando di conservare il suo potere di dignitario, scatena l'insana gelosia di Gianciotto, conducendo tutti i protagonisti della storia all'epilogo finale.

Soltanto questa parte del film procede con una approssimazione al Canto dantesco: è la scena in cui l'amore compresso di Paolo e Francesca si dichiara esplicitamente davanti al Libro galeotto; si guardano in viso mentre leggono la storia di Lancillotto e Ginevra e finalmente si danno un bacio. Ma Francesca si ritrae subito dopo, consapevole del rischio corso per l'amato Paolo e del peccato appena commesso. Poi il finale tragico, e qui il riferimento a Dante si complica con una precisa allusione al dramma shakespeariano di Giulietta e Romeo. Un cinico intrigo ordito dall'astrologo conduce i tre, Gianciotto, Paolo e Francesca, allo scontro mortale: dapprima Francesca si fa consegnare dall'astrologo una piccola boccia di veleno, e nell'incontro finale con Paolo tenta vanamente di farlo partire, per salvarlo dall'ira del fratello, e poi uccidersi; ma Paolo si rende conto del suo progetto e resta con Lei, quando sopraggiunge Gianciotto che, avuta visibile conferma del sospetto istillato dall'astrologo, con un pugnale colpisce a morte i due amanti - a questo punto si potrebbe dire: innocenti, essendo non realmente colpevoli se non nel proprio sentimento. Prima di spirare, Francesca pronuncia a Gianciotto (che arretra come inorridito dalle conseguenze del suo gesto) parole definitive: "ascolta... volevamo andar via insieme... questo è il nostro convegno di morte e questo - consegnando allo stesso Gianciotto la boccetta di veleno - il viatico...” . 


\section{Il dRamma FILMico SECONdo Matarazzo}

Nel finale, Matarazzo ricompone diverse linee del plot: l'amore dei due giovani confligge con le esigenze pubbliche che danno a Gianciotto, fratello di Paolo e sovrano di Rimini, una moglie che - pur amando segretamente, e da sempre, il cognato - è tuttavia fedele alla promessa del matrimonio, da lei forzatamente subito. Chi ha esperienza della filmografia di Matarazzo riconosce in questa impostazione drammatica ciò che rende i suoi film accumuli di passioni, dapprima fortemente controllate e poi, soltanto in pochi punti della storia, lasciate libere di esprimersi. Che è quel che accade nella scena del Libro galeotto, dove tuttavia l'andamento del film dispone qualcosa di ben diverso dal canto dantesco. Ciò perché, se nell'opera di Dante i due amanti scoprono di esser tali solo nella complicità (sentimentale, e tuttavia inequivocabilmente "mediale") del Libro, nel film di Matarazzo lo spettatore sa invece che, davanti al medium galeotto, si verifica qualcosa di già preesistente e ben noto.

Paolo e Francesca, nel film, attorno al Libro sanciscono qualcosa di avvenuto tempo prima, e questo incontro è soltanto (formalmente, e dunque conclusivamente) la realizzazione di una tensione fino ad allora contratta e protratta e che, davanti al Libro, si scioglie e si libera. $\mathrm{Ma}$ - ed è questo il punto - per volgersi in una direzione affatto negativa e mortale. Ecco che, come in una svolta repentina, il dramma acquista una visualizzazione fosca e forse addirittura "gotica".

Tranne che nella prima parte - l'incontro fra i due giovani in una Ravenna assediata ma al chiaro della luce diurna - il film, difatti, prende la piega di un racconto ambientato in architetture medioevali dai toni claustrali, spesso chiaroscurali, con diversi riferimenti visivi a una tradizione illustrativa sedimentata in Italia fra Otto e Novecento (basti pensare a disegnatori come Gustavino o Rino Albertarelli e ai loro, similari, Faust, che paiono essere ben noti a Matarazzo, in quanto dalle loro tavole disegnate [Figg. I-2] sembrano essere chiaramente ripresi l'abbigliamento e il copricapo con cui visivamente è contraddistinto l'animo astuto e maligno dell'astrologo - molto ben interpretato da Aldo Silvani [Fig. 3]). ${ }^{5}$

5. Questo film di Matarazzo non sembra riconoscere alcun debito, se non generico, con le illustrazioni di Gustave Doré, autore che invece è direttamente citato, con alcune sue immagini, nei titoli di testa de Il conte Ugolino di Freda. Occorre ribadire che Doré - all'universo visivo del quale si rifanno diversi registi nei film muti e in qualche film sonoro - resta autore caposaldo della visualizzazione dell'opera dantesca, la cui importanza e la cui diffusione almeno fra Ottocento e Novecento - è talmente vasta che, alla fine, anche quando egli non è esplicitamente citato, l'influenza esercitata è praticamente extra-testuale. Forse l'influenza di Doré la si può dire, proprio nel doppio senso tecnico e generale, contestuale o culturale. $\mathrm{Ma}$, nel caso del film di Matarazzo non c'è se non un'ombra molto lieve dell'influenza di Doré, mentre altri illustratori - nei segnali e negli elementi sopra rilevati - sono tenuti in forte considerazione. 
Nella parte che precede l'epilogo, Matarazzo compone un tragitto narrativo irreversibile, che conduce i protagonisti all'esito tragico. Le azioni del fosco astrologo sono doppie nella menzogna agita alle spalle di Paolo e Francesca; la fiducia di Gianciotto nei confronti della moglie è rosa dal dubbio istillato, senza nemmeno eccessiva pressione, dall'astrologo; il desiderio di Francesca di terminare la propria vita e dunque chiedere all'astrologo una boccetta di veleno (rovesciando di segno l'espediente shakespeariano di Giulietta e Romeo) è però usato dall'astrologo per esasperare il sospetto di Gianciotto che vi sia una trama contro di lui.

Tutto questo rende l'epilogo davvero efficace nella propria evoluzione drammatica, e molto conseguente alle premesse: il convegno d'amore - in tal modo inteso, malamente, da Gianciotto, che reagisce contro il fratello Paolo e contro Francesca con il gesto del duplice assassinio (visivamente situato da Matarazzo nell'immagine dello schermo nero, ossia in quel fuori campo che lascia allo spettatore il compito di vedere, e sentire, nella propria immaginazione l'effettuarsi del colpo di pugnale) - si rivela essere un convegno di morte, e la speranza di felicità tenuta ben nascosta nel proprio cuore dai due giovani è capovolta in una - quasi efferata - liberazione dal mondo terreno.

L'andamento di questo finale tragico, per quanto annunciato dalla conoscenza del testo dantesco, rivela una soggiacente impostazione teatrale. $\mathrm{Si}$ tratta, tuttavia, di un teatro assunto nella cifra ben dinamica e attiva di un cinema drammatico senza sconti e senza scappatoie. Matarazzo punta a un cinema che recuperi del teatro la sostanza emozionale più profonda: non fatta di movenze recitative, e nemmeno di dialoghi impostati e vocalizzati, bensì di calibrati effetti scenici, e del senso ineluttabile di azioni (ecco il proprio del cinema narrativo!..) che comportino profonde ripercussioni dell'anima.

Nell'ultimissima sequenza, quando Matarazzo accomiata lo sguardo degli spettatori dalla scena dei due amanti, per terra nella camera in cui sono stati uccisi, attraverso un carrello indietro che si allontana da loro qualche secondo prima della Fine, e prima che una porta chiuda per sempre la scena dei due amanti caduti nella Morte, in trasparenza si leggono i tre ben noti capoversi del Canto dantesco: Amor, ch'al cor gentil ratto s'apprende; Amor, ch'a nullo amato amar perdona; Amor, condusse noi ad una morte. In tal punto, la corrispondenza fra il film e i versi danteschi, in maniera appropriata fino a quel momento tenuta alla larga, perfino evitata secondo una strategica scelta di eliminarla dapprincipio, torna perentoriamente, ribadendo l'equivalenza intima, emozionale, fra i versi del Canto $\mathrm{V}$ e le immagini filmiche.

La fortuna dei versi di Dante può, in questo modo, considerarsi rigenerata e, nel contempo, prolungata nel dramma e nella visione di Matarazzo. 


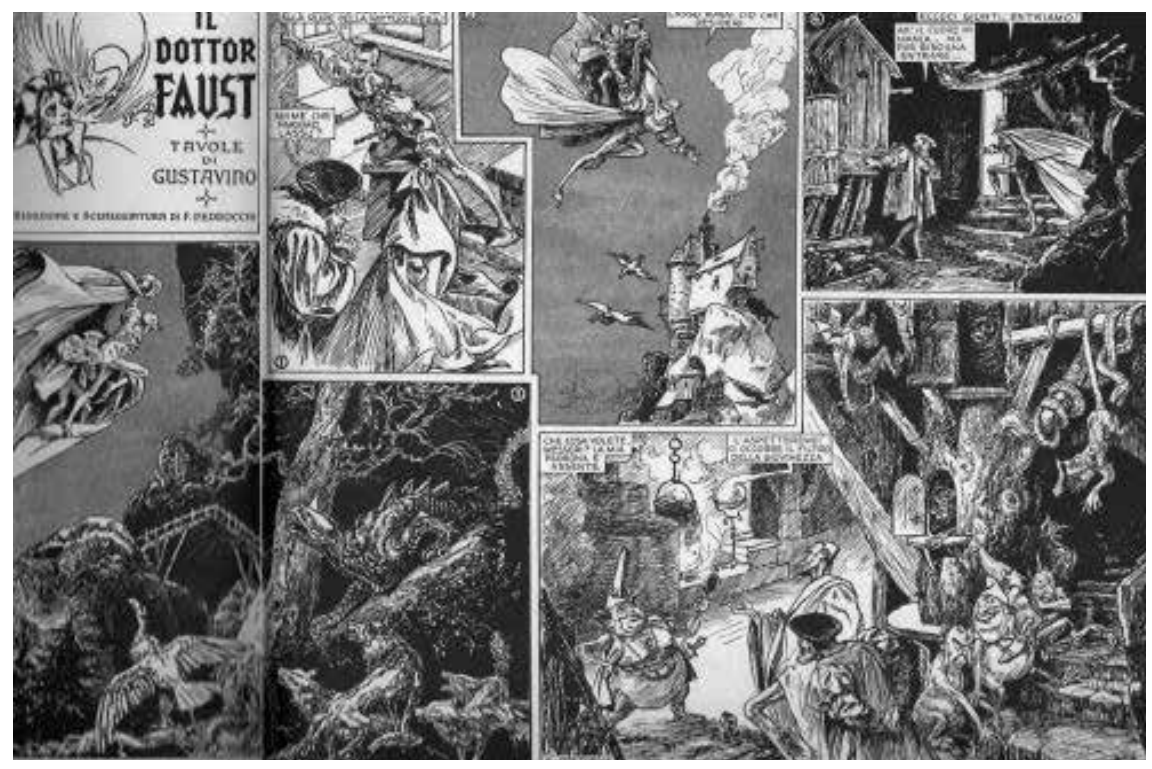

Fig. I: Gustavino, tavola da Faust,1939.
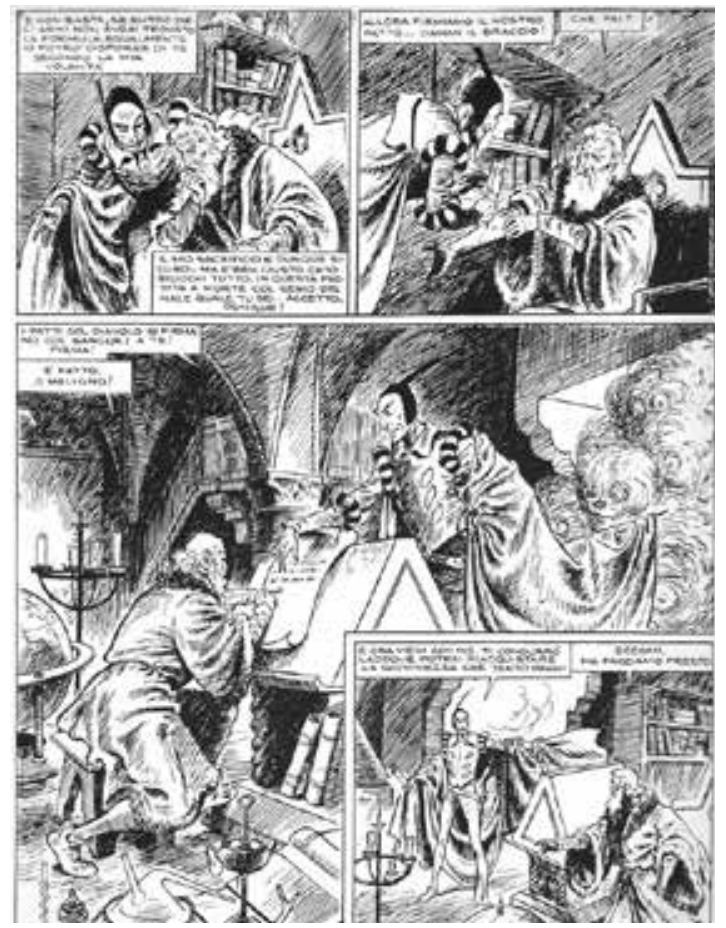

Fig. 2: Rino Albertarelli, tavola da Faust, 194I. 


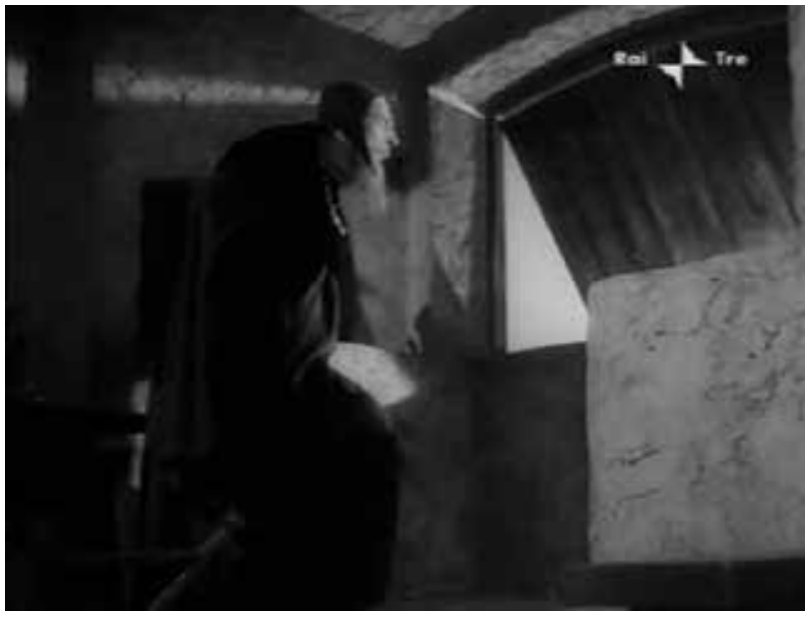

Fig. 3: Il personaggio dell'astrologo di Paolo e Francesca di Matarazzo.

\section{RIFERIMENTI BIBLIOGRAFICI}

Aprà, A., Carabba, C., 1976, (a cura di), Neorealismo d'appendice. Per un dibattito sul cinema popolare: il caso Matarazzo, Rimini-Firenze, Guaraldi.

Aprà, A., Freccero, C., Grasso, A., Grek Germani, S., Lombezzi, M., Pistagnesi, P., Sanguineti, T., I976, (a cura di), Raffaello Matarazzo. Materiali, Torino, Quaderni del Movie Club.

AA.VV., I980, Neorealismo. Poetiche e polemiche, (a cura di Claudio Milanini), Milano, Il Saggiatore.

AA.VV., I985, Cinecittà I: Industria e mercato nel cinema italiano tra le due guerre, Venezia, Marsilio.

Casetti, F., Fanchi M. G., 2006, (a cura di), Terre incognite. Lo spettatore italiano e le nuove forme dell'esperienza di visione del film, Roma, Carocci.

Casetti, F., Mosconi, E., 2006, (a cura di), Spettatori italiani. Riti e ambienti del consumo cinematografico (I900-I950), Roma, Carocci.

Deleuze, G., 1984, L'immagine movimento, Milano, Ubu Libri

—, I989, L'immagine tempo, Milano, Ubu Libri.

Della Casa, S., 200o, Cinema popolare italiano del dopoguerra, in Brunetta, G. P., (a cura di), Storia del cinema mondiale, L'Europa. Le cinematografie nazionali, vol. III, Torino, Einaudi, pp. 779-823.

Freda, R., I98I, Divoratori di celluloide. so anni di memorie cinematografiche e non, Milano, Emme Edizioni-Il Formichiere.

Frezza, G., 1996, Le disomogeneità strutturali del cinema italiano, in Frezza, G., Cinematografo e Cinema. Dinamiche di un processo culturale, Bologna, Cosmopoli, pp. 97-I24.

Magrelli, E., I986, (a cura di), Cinecittà 2. Sull'industria cinematografica italiana, Venezia, Marsilio

Parigi, S., 20I4, Neorealismo. Il nuovo cinema del dopoguerra, Venezia, Marsilio.

Zagarrio, V., I988, (a cura di), Cinecittà 3. Dietro lo schermo. Ragionamenti sui modi di produzione cinematografici in Italia, Venezia, Marsilio. 